\title{
Lifetime improvement of green phosphorescent organic light-emitting diodes by charge confining device structure
}

\author{
Sung Hyun Kim and Jyongsik Jang \\ School of Chemical and Biological Engineering, Seoul National University, Shinlim-dong, Kwanak-gu, \\ Seoul 151-742, Korea \\ Jun Yeob Lee ${ }^{\mathrm{a})}$ \\ Department of Polymer Science and Engineering, Dankook University, Hannam-dong, Yongsan-gu, \\ Seoul 140-714, Korea
}

(Received 5 April 2007; accepted 25 April 2007; published online 17 May 2007)

\begin{abstract}
Lifetime improvement of green phosphorescent organic light-emitting diodes by charge confinement inside an emitting layer was investigated. Excitons were confined within the emitting layer by using a charge confining structure with a high doping concentration at the center of the emitting layer. The lifetime of green devices could be improved by more than five times by confining the excitons at the center of the emitting layer. (C) 2007 American Institute of Physics. [DOI: 10.1063/1.2740587]
\end{abstract}

Phosphorescent organic light-emitting diodes (PHOLEDs) have been actively studied for the past ten years due to their merit of high quantum efficiency. $100 \%$ internal quantum efficiency can be realized in PHOLEDs because both singlet and triplet excitons can contribute to light emission. ${ }^{1}$ However, the lifetime of PHOLEDs needs to be improved further even though red PHOLEDs are already commercialized.

Lifetime is one of the critical issues of PHOLEDs, but there have not been many studies to improve the lifetime of PHOLEDs. One approach to get a long lifetime of PHOLEDs was to develop, stable host, dopant and hole blocking materials. ${ }^{2-4}$ Kwong et al. compared the lifetime of several hole blocking materials and reported that biphenoxybi(8-hydroxy-3-methylquinoline) aluminum (Balq) is better than other hole blocking materials in terms of lifetime. ${ }^{2}$ Kwong et al. group also demonstrated the long lifetime of red and green PHOLEDs by using stable dopant materials. ${ }^{3}$ Electron-transport-type host material was also effective to improve the lifetime of PHOLEDs and the long lifetime could be obtained by using thermally stable host materials. ${ }^{4}$ The other approach to get a stable lifetime of PHOLEDs was to apply a device architecture which can give a stable lifetime. Our group studied the lifetime of green PHOLEDs with a graded doping structure and reported that a graded doping structure was beneficial to get a long lifetime of PHOLEDs. ${ }^{5}$ The lifetime of green PHOLEDs could be enhanced by more than three times by using a graded doping structure which has a high doping concentration near the hole transport layer.

In this work, the lifetime of green PHOLEDs was investigated by using a charge confining structure with a high doping concentration at the center of the emitting layer. The lifetime of charge confining devices was compared with that of standard devices.

The device configuration used in this experiment was indium tin oxide (ITO, $150 \mathrm{~nm}$ )/ $N, N^{\prime}$-diphenyl-150 $\mathrm{nm}) / N, N^{\prime}$-bis-[4-(phenyl-m-tolyl-amino)-phenyl]-biphenyl4,4'-diamine (60 nm / $N, N^{\prime}$-di(1-naphthyl)- $N, N^{\prime}$-diphenylbenzidine (NPB, $30 \mathrm{~nm}) /$ light-emitting layer(EML, $30 \mathrm{~nm}) /$

\footnotetext{
a) Author to whom correspondence should be addressed; Fax: 82-2-709-
} 2614; electronic mail: leej17@dankook.ac.kr
Balq $\quad(5 \mathrm{~nm}) / \quad$ tris $(8$-hydroxyquinoline $) \quad$ aluminum $\left(\mathrm{Alq}_{3}, 25 \mathrm{~nm}\right) / \mathrm{LiF}(1 \mathrm{~nm}) / \mathrm{Al}(200 \mathrm{~nm})$. Three devices were fabricated to investigate the effect of the device structure on lifetime. Two standard devices with single EML and one device with three EMLs were prepared. The total thickness of the EML was constant for all devices. Standard devices which have $\left(4,4{ }^{\prime}-N, N^{\prime}\right.$-dicarbazole)biphenyl (CBP) and PH1 as hosts for the EML and a charge confining device with PH1 EML sandwiched between CBP EMLs were fabricated. A dopant material was tris(2-phenylpyridine) iridium $\left[\operatorname{Ir}(\mathrm{ppy})_{3}\right]$ and doping concentrations were $5 \%$ and $9 \%$ for CBP and PH1. PH1 was supplied from Merck Co. and it has a spirobifluorene-type backbone structure with high electron transport properties. The triplet band gap of PH1 was $2.4 \mathrm{eV}$ and the highest occupied molecular orbital and the lowest unoccupied molecular orbital (LUMO) were 5.9 and $2.8 \mathrm{eV}{ }^{6}$

ITO glass substrates were cleaned with acetone and isopropyl alcohol in an ultrasonic bath for $15 \mathrm{~min}$, respectively, and were dried at $120{ }^{\circ} \mathrm{C}$ for $2 \mathrm{~h}$ before use. The ITO glass substrates were exposed to UV ozone for $15 \mathrm{~min}$ and were transferred to an evaporation chamber. Organic materials were deposited at a deposition rate of $1 \AA$ /s and the doping concentration was controlled by changing the deposition rate of dopant materials. LiF was deposited at a rate of $0.1 \AA / \mathrm{s}$ and Al was evaporated at a rate of $5 \AA / s$. The organic materials, $\mathrm{LiF}$ and $\mathrm{Al}$, were deposited at a base pressure of 5 $\times 10^{-7}$ torr. After cathode deposition, the devices were encapsulated with a glass lid and calcium oxide getter. Lifetime measurements were carried out at a constant current condition from an initial luminance of $2000 \mathrm{~cd} / \mathrm{m}^{2}$ using photodiode and Keithley 2400 source measurement unit. Currentdensity-voltage-luminance characteristics were studied by using Keithley 236 source measurement unit and PR 650 spectrophotometer.

A charge confining structure of OLEDs can provide high efficiency because holes and electrons can be confined inside the EML, as reported by our group. ${ }^{6}$ The high efficiency of OLEDs can be beneficial to device stability because the device would be operated at a low electrical stress. In addition, it can be expected that the lifetime can be improved by a charge confining structure as the hole and electron leakage out of the EML can be minimized. ${ }^{7}$ In this work, a charge 
(a)
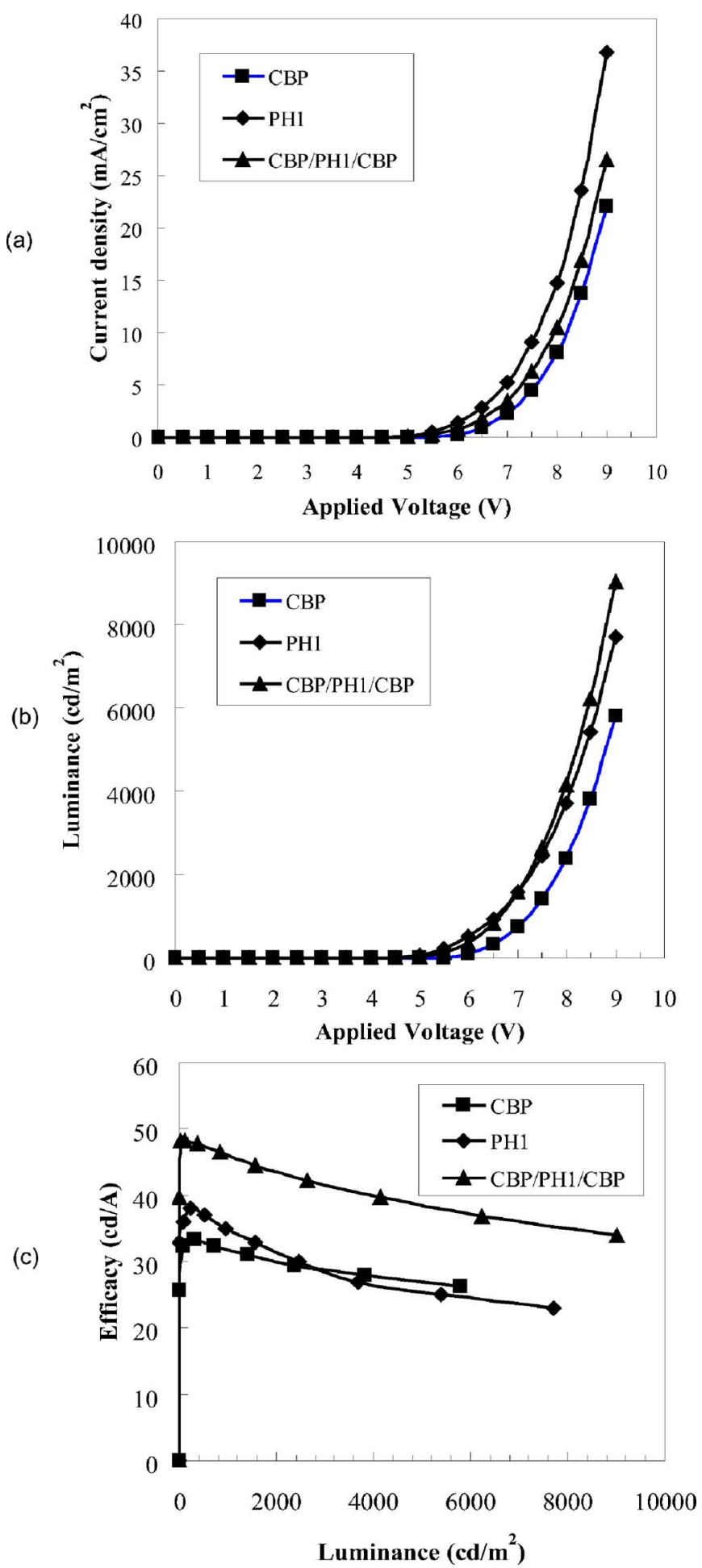

FIG. 1. Current-density-voltage-luminance curves of standard devices and charge confining device. (a) Current-density voltage, (b) luminance voltage, and (c) efficacy luminance.

confining structure which can confine charges at the center of the light-emitting layer was developed to enhance the lifetime of green PHOLEDs. A high doping concentration was used at the center of the emitting layer (PH1 layer) and a normal doping concentration was applied in CBP layers. The lifetime of a charge confining device was compared with that of CBP and PH1 standard devices.

Current-density-voltage-luminance characteristics of charge confining devices are shown in Fig. 1. Optimized doping concentrations of 5\% and 9\% for CBP and $\mathrm{PH} 1$ were Downloaded 23 Nov 2007 to 147.46 .143 .55 . Redistribution subject

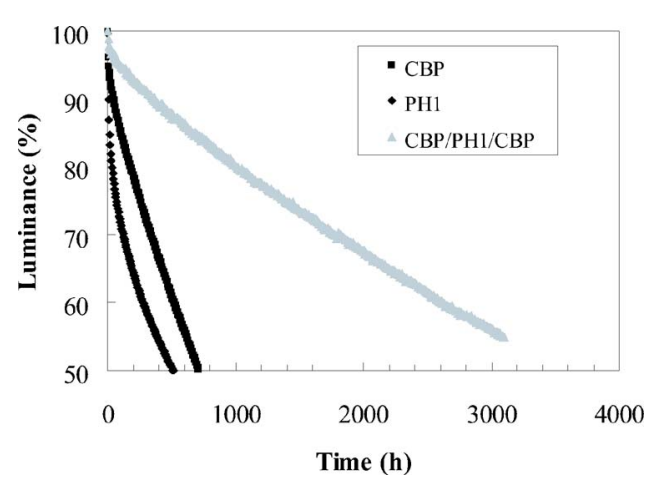

FIG. 2. Lifetime of the multilayer charge confining device compared with that of standard devices at an initial luminance of $2000 \mathrm{~cd} / \mathrm{m}^{2}$.

applied for all devices. The current density was high in the PH1 device, while the CBP device showed the lowest current density. The high current density of the PH1 device is due to the efficient electron injection and high doping concentration. PH1 has a LUMO value of $2.8 \mathrm{eV}$ and an electron injection from Balq to PH1 is very efficient. In addition, $\mathrm{PH} 1$ has a spirobifluorene backbone structure, facilitating electron transport inside the EML. A high doping concentration in the PH1 device is also responsible for the high current density. The rather low current density of the charge confining device is mainly due to the electron trapping in the PH1 layer. Luminance showed almost the same trend as current density and a luminance efficacy was calculated from current density and luminance. The luminance efficacy was improved by $50 \%$ by using a charge confining structure, which is attributed to the charge confinement in the light-emitting layer. Hole transport is retarded in the PH1 layer and electron can be trapped in the PH1 layer due to the low lying LUMO level of $2.8 \mathrm{eV}$ of PH1. Therefore, charge can be confined inside the emitting layer, enhancing hole and electron charge balance.

Figure 2 shows the lifetime of a triplet quantum well device compared with that of standard devices. The lifetime was measured under constant current condition from an initial luminance of $2000 \mathrm{~cd} / \mathrm{m}^{2}$ and the lifetime of charge confining devices could be improved by more than five times compared with that of standard PH1 and CBP devices. The lifetime of the charge confining device was $3500 \mathrm{~h}$, while it was $570 \mathrm{~h}$ in the PH1 device and $690 \mathrm{~h}$ in the CBP device. The extended lifetime of the charge confining device can be explained by high efficacy and charge confinement inside the EML. The efficacy of the charge confining device was $43 \mathrm{~cd} / \mathrm{A}$, while it was 31 and $30 \mathrm{~cd} / \mathrm{A}$ in the PH1 and CBP devices. High efficiency can have a positive effect on the lifetime because the device can be operated at a low current density. However, a $40 \%$ improvement of efficacy in the charge confining device cannot explain the five times increase of the lifetime. Charge confinement in the lightemitting layer may additionally increase the lifetime of PHOLEDs. It is well known that hole transport to the $\mathrm{Alq}_{3}$ layer degrades device stability due to the unstable $\mathrm{Alq}_{3}$ cation formation. ${ }^{7}$ In general, electron transport material is stable under electron injection rather than hole injection, while hole transport material is stable under hole injection rather than electron injection. In a poly(2,5-ethylenedioxyythiophene):polystyrenesulfonate (PEDOT:PSS) device, it was reported that electron injection from polymer emitter to PEDOT:PSS was responsible for the to AIP license or copyright; see http://apl.aip.org/apl/copyright.jsp 


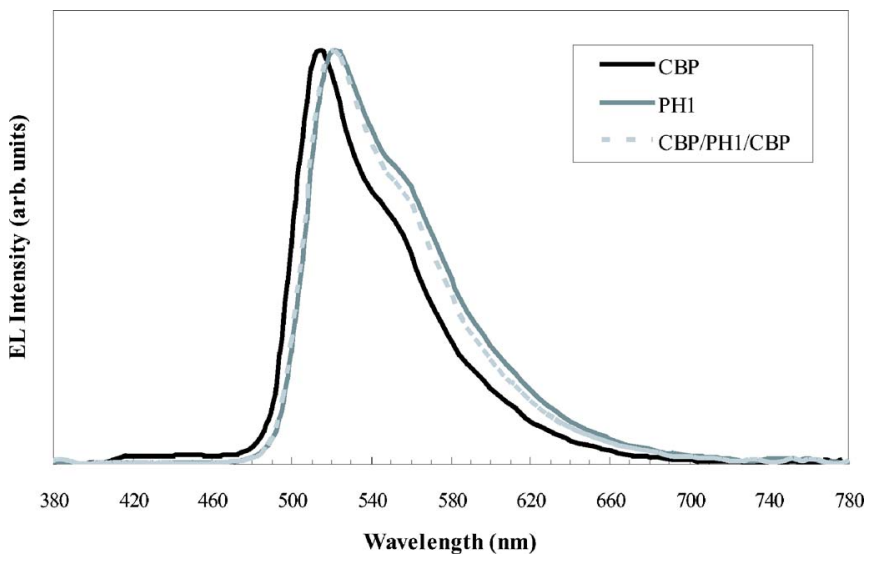

FIG. 3. Electroluminescence spectra standard devices and charge confining devices.

short lifetime of polymer devices. ${ }^{8,9}$ Therefore, charge carrier confinement inside the light-emitting layer may increase the lifetime of PHOLEDs without damaging the charge transport layer. The charge confinement inside the emitting layer can be proved from electroluminescence (EL) spectra of standard devices and charge confining devices.

To confirm the relationship between the charge carrier confinement inside the light-emitting layer and the lifetime of PHOLEDs, EL spectra of charge confining devices were compared with those of standard devices. Figure 3 shows EL spectra of three devices fabricated in this work. PH1 and CBP standard devices exhibited main emission peaks from $\operatorname{Ir}(\text { ppy })_{3}$ at 520 and $516 \mathrm{~nm}$, respectively, and the charge confining device showed a peak at $520 \mathrm{~nm}$. EL spectrum of the charge confining device was similar to that of PH1 except for a small blueshift of a shoulder peak around $550 \mathrm{~nm}$. This result indicates that most of the light emission in the charge confining device originated from the $\mathrm{PH} 1$ emission rather than from the CBP emission. Holes are effectively transported from the CBP layer to the PH1 layer at a high doping concentration because hole hopping between dopant sites in the PH1 layer is facilitated at a high doping concentration. Even though the hole injection from the CBP layer to the $\mathrm{PH} 1$ layer is facilitated at a high doping concentration, holes cannot be efficiently transported from the PH1 layer to the next CBP layer. A low doping concentration in the CBP layer and poor hole transporting properties of $\mathrm{PH} 1$ limit the hole transport inside the emitting layer. Therefore, holes can be confined in the PH1 layer and most electrons would stay in the PH1 layer due to the electron injection energy barrier between $\mathrm{PH} 1$ and $\mathrm{CBP}$, resulting in the exciton formation inside the PH1 layer. A small emission peak around $450 \mathrm{~nm}$ in the CBP device is due to the NPB emission, which is attributed to the electron overflow from the CBP layer to NPB. ${ }^{10}$ The NPB emission in the CBP device was not detected in PH1 and charge confining device. The disappearance of the NPB emission in the charge confining device is due to the charge trapping in the PH1 layer. NPB emission may have a negative effect on lifetime because the lightemitting efficiency of NPB is quite low ${ }^{11}$ and hole-transporttype materials are unstable under electron attack. ${ }^{7}$ It was found in our work that blocking of NPB emission improves device stability under the same current density, which will be published later. ${ }^{12}$

In summary, the lifetime of green PHOLEDs could be improved by more than five times by using a triplet exciton confining device structure. The lifetime enhancement mechanism of quantum well devices was investigated with EL spectra during lifetime measurement and it was found that the triplet exciton confinement inside the emitting layer was responsible for long lifetime of charge confining devices.

${ }^{1}$ C. Adachi, M. Baldo, M. E. Thompson, and S. R. Forrest, J. Appl. Phys. 90, 5048 (2001).

${ }^{2}$ R. C. Kwong, M. R. Nugent, L. Michalski, T. Ngo, K. Rajan, Y. Tung, M. S. Weaver, T. X. Zhou, M. Hack, M. E. Thompson, S. R. Forrest, and J. J. Brown, Appl. Phys. Lett. 81, 162 (2002).

${ }^{3}$ R. C. Kwong, M. S. Weaver, M. M. Lu, Y. Tung, A. B. Chang, T. X. Zhou, M. Hack, and J. J. Brown, Org. Electron. 4, 155 (2003).

${ }^{4}$ H. Becker, H. Vestweber, A. Gerhard, P. Stoessel, H. Heil, and R. Fortte, SID Int. Symp. Digest Tech. Papers 36, 1062 (2005).

${ }^{5}$ J. Y. Lee, Appl. Phys. Lett. 89, 153503 (2006).

${ }^{6}$ S. H. Kim, J. Jang, and J. Y. Lee, Appl. Phys. Lett. 90, 173501 (2007).

${ }^{7}$ H. Aziz, Z. D. Popovic, N.-X. Hu, A.-M. Hor, and G. Xu, Science 283, 1900 (1999).

${ }^{8}$ T.-W. Lee, O. Kwon, M.-G. Kim, S. H. Park, J. Chung, S. Y. Kim, Y. Chung, J.-Y. Park, E. Han, D. H. Hur, J.-J. Park, and L. Pu, Appl. Phys. Lett. 87, 231106 (2005)

${ }^{9}$ J. H. Burroughes, Asia Display/IMID'2004, Daegu, Korea, 24-27 August 2004 (unpublished).

${ }^{10}$ M. Ikai, S. Tokito, Y. Sakamoto, T. Suzuki, and Y. Taga, Appl. Phys. Lett. 79, 156 (2001).

${ }^{11}$ M. Kinoshita, H. Kita, and Y. Shirota, Adv. Funct. Mater. 12, 780 (2002).

${ }^{12}$ J. Y. Lee (unpublished). 